\title{
AN INTEGRATIVE APPROACH TO ANALYZE EEG SIGNALS AND HUMAN BRAIN DYNAMICS IN DIFFERENT COGNITIVE STATES
}

\author{
Jeffrey Jonathan (Joshua) Davis ${ }^{1}$, Chin-Teng Lin ${ }^{2}$, Grant Gillett ${ }^{3}$, and Robert Kozma ${ }^{4}$ \\ ${ }^{1}$ The Embassy of Peace, Whitianga, New Zealand, e-mail: joshua_888@yahoo.com \\ ${ }^{2}$ Centre of Artificial Intelligence, Faculty of Engineering and Information Technology \\ University of Technology Sydney, Australia, e-mail: chintenglin@gmail.com \\ ${ }^{3}$ Department of Bioethics, University of Otago, Dunedin, New Zealand, \\ e-mail: grant.gillett@stonebow.otago.ac.nz. \\ ${ }^{4}$ Department of Mathematical Sciences, University of Memphis, TN \\ and University of Massachusetts Amherst, MA, USA, \\ e-mail: rkozma@cs.umass.edu
}

Submitted: 25th February 2017; accepted: 14th April 2017

\begin{abstract}
Electroencephalograph (EEG) data provide insight into the interconnections and relationships between various cognitive states and their corresponding brain dynamics, by demonstrating dynamic connections between brain regions at different frequency bands. While sensory input tends to stimulate neural activity in different frequency bands, peaceful states of being and self-induced meditation tend to produce activity in the mid-range (Alpha). These studies were conducted with the aim of: (a) testing different equipment in order to assess two (2) different EEG technologies together with their benefits and limitations and (b) having an initial impression of different brain states associated with different experimental modalities and tasks, by analyzing the spatial and temporal power spectrum and applying our movie making methodology to engage in qualitative exploration via the art of encephalography. This study complements our previous study of measuring multichannel EEG brain dynamics using MINDO48 equipment associated with three experimental modalities measured both in the laboratory and the natural environment. Together with Hilbert analysis, we conjecture, the results will provide us with the tools to engage in more complex brain dynamics and mental states, such as Meditation, Mathematical Audio Lectures, Music Induced Meditation, and Mental Arithmetic Exercises. This paper focuses on open eye and closed eye conditions, as well as meditation states in laboratory conditions. We assess similarities and differences between experimental modalities and their associated brain states as well as differences between the different tools for analysis and equipment.
\end{abstract}

Keywords: cognition, EEG, analytic amplitude, analytic phase, Hilbert transform, visual cortex, consciousness, meditation, emotions, awareness, intentionality, spiritual values 


\section{Introduction}

The human brain is a complex system that interacts and communicates on a moment to moment basis with a variety of other systems in the body, such as the nervous, cardiovascular and respiratory systems, all of which combine to form an integrated whole or a larger, integrated system - the human body. The above suggests that any change that appears in one part of the body affects, either directly or indirectly, all the other parts of the body. The brain plays an essential role in evaluating, coordinating and controlling human behavior and in particular, intentional action and decision making and thus, the study of brain dynamics offers a valuable approach to better understand and gain insight into the possibility of generating peaceful and harmonious states of being at will as is done in most meditation practices.

While sensory input tends to stimulate neural activity in different frequency bands $[1,3]$, peaceful states of being and self-induced meditation tend to produce activity in the mid-range (Alpha) [2, 6, $25,26,27,28,29]$. In this study we aim at characterizing different cognitive states mathematically, statistically or geometrically, as an initial and preliminary attempt to discriminate between them. In the long term we aim at developing a methodology and set of tools that would allow us to better understand the differences between stress-associated cognitive states and peace-associated ones.

The developed tools help us to engage in more complex and thorough analysis of brain dynamics in different cognitive states, like Meditation, Mathematic Audio Lectures, Music Induced Meditation, and Mental Arithmetic Exercises. This paper will focus on the modalities of closed eye (CE), open eye $(\mathrm{OE})$, open eye with flashlight (OEFL) and meditation (MED) in laboratory conditions in order to assess similarities and differences between experimental modalities and their associated brain states as well as differences between the different tools for analysis and equipment.

In the first study we used an electrode array prototype MINDO-48S-001AFF0900A7, produced by BRC/NCTU, Hsinchu, Taiwan (Liao et al., 2012; Kozma et al., 2013). MINDO-48S oversamples EEG+EMG via 48 closely spaced spring-loaded dry electrodes attached to a flexible curvilinear band that could be quickly fixed on the scalp of a volunteer in any orientation. The electrodes had a length of $3 \mathrm{~mm}$ and diameter of $1 \mathrm{~mm}$, and they use a special golden alloy. Also, MINDO-48S uses wireless transmission through Bluetooth to communicate with a laptop computer, which serves as portal with GUI and also saves the measured data.

In this research, brain activity of two (2) female human volunteers was recorded using a Mitsar EEG cap of 19 gel-electrodes [13]. While participants lay comfortably in a relaxed mode, their brain activity was measured in four different modalities: (1) with closed eyes, (2) with open eyes, (3) with open eyes and a visual stimuli (intermittent flashlight), and (4) meditating with the intention of generating peaceful states of being. These were the same two (2) participants of a previous study, where the same modalities, apart from the one of meditation were measured with a different equipment and technology [11].

The main goal of these studies was to apply signal processing techniques and a movie making methodology similar to [23], that could support the challenging task of distinguishing and differentiating between different brain dynamics and the four above mentioned conditions, towards a deeper understanding of neural correlates of consciousness (NCC) $[8,9,10,11,12,20,22]$. This initial research, we hope, suggests a direction to approach the exploration of questions in regards to consciousness, mental activity and how it reflects in brain dynamics.

\section{Description of Equipment}

For a detailed description of the equipment used in the first study, see [11]. Experiments have been completed according IRB protocol \#13.10.0020 (Liberty IRB, expiration: Oct. 8, 2015). For the purpose of illustration and comparison, the reader can observe a picture of the MINDO-48-S array in Figure 1. For the second study, the electroencephalograph (EEG) hardware used in these experiments was the Mitsar 201 electro-cap containing 19 gel-electrodes. The cap is made from "an elastic spandex-type fabric with recessed, pure tin electrodes attached to the fabric." The cap covers 
the whole scalp and "electrodes are positioned to the International 10-20 method of electrode placement." [13]
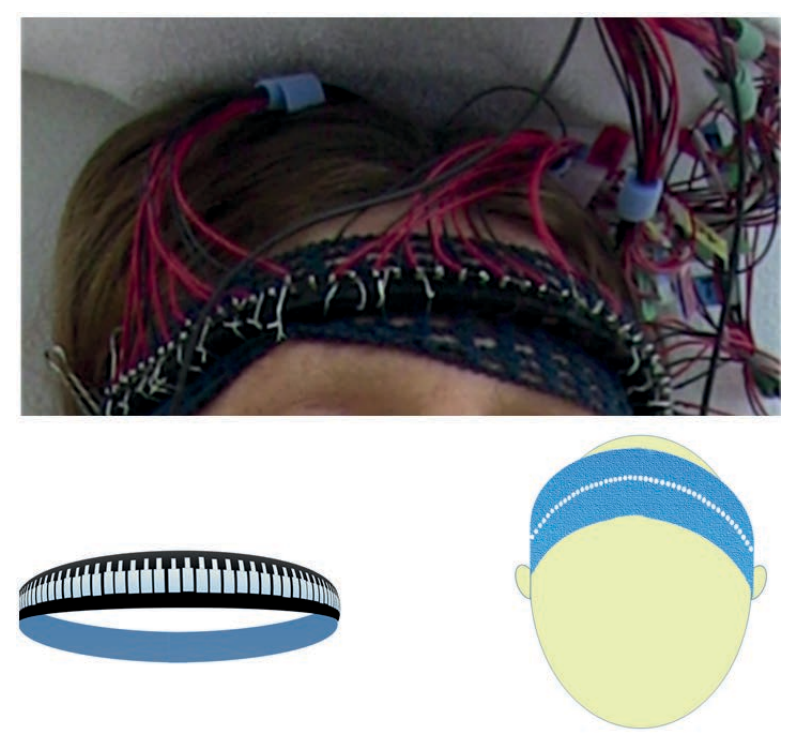

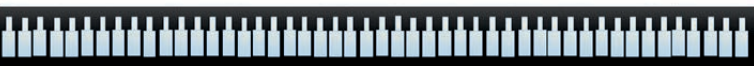

123456789

Figure 1. MINDO-48-S headband on the forehead of a participant with a graphical description of the 48 spring loaded set of dry electrodes. Top: Participant's forehead covered with the electrode band, Middle left: headband view from the inside, Middle right: headband placed with electrodes towards the forehead, Bottom: rubber band with electrodes detached from the headband.
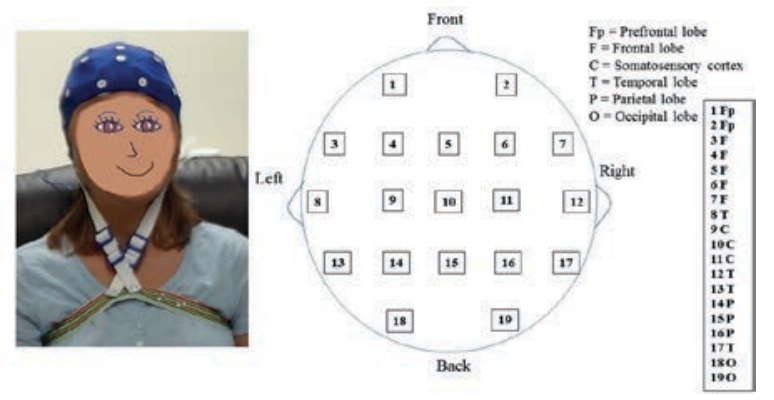

Figure 2. Shows a picture of the cap side by side with a map showing where the electrodes were placed: $\mathrm{Ch} 1 \& \mathrm{Ch} 2$ are labeled Prefrontal lobe

(Fp); Ch3-Ch7 Frontal lobe (F); Ch9-Ch11

Somatosensory Cortex (C); Ch8, Ch12-Ch13,

Ch17 Temporal lobe (T); Ch14-Ch15 Parietal lobe (P); Ch18 \& Ch19 Occipital lobe (O).
Experiments were performed with two (2) human participants and one (1) control object without neural activity (a soccer ball), and were conducted for duration of seven (7) minutes, each experiment being performed twice. To ensure a clear reading of the signal, participants were trained to minimize artifacts from blinking and other bodily movements as the previous study explains. After measuring, notch filters were applied to signals to remove the $60 \mathrm{~Hz}$ frequency artifacts created by electrical influences. The sampling frequency was $500 \mathrm{~Hz}$ as specified in [24].

\section{Description of Experiments}

Following we define the four (4) modalities we considered for these experiments in general and the particularities for this new study.

Modality 1 - Closed Eyes (CE) - The objective of this experiment was to measure brain activity in different areas of the brain while the participant lay on her back with eyes closed in a relaxed state for duration of seven (7) minutes.

Modality 2 - Open Eyes (OE) - The objective of this experiment was to measure brain activity in different areas of the brain while the participant lay on her back with eyes open and minimal blinking for duration of seven (7) minutes.

Modality 3 - Open Eyes with Flash Light (OEFL) -The objective of this experiment was to measure brain activity in different regions of the brain as the participant lay on her back with eyes open and a flashlight was held about $60 \mathrm{~cm}$ from her forehead. The light flashed intermittently at a relatively high frequency throughout the duration of the experiment ( 7 minutes). As in modality 2 the participant intentionally aimed to blink minimally.

Modality 4 - Meditation (MED) - The objective of this experiment was to measure brain activity in different regions of the brain as the participant lay on her back with closed eyes in a meditative state for 7 minutes.

\section{Experimental Procedures}

For this new study, experiments took place in a lab setting where the participant lay on a mattress covered by a duvet resting her head on a folded towel 
or pillow. The experiments were performed during the day with no lights on and curtains were drawn to create a minimal light atmosphere together with a relatively stable room temperature. All electrical devices in the lab were switched off or ran on battery power. In addition to the EEG system, other equipment used included a working table, laptop PC, iPhone and thermometer. The participant's pulse and the room temperature were recorded at the start and finish of experiments. Morning or afternoon sessions ran for approximately 2-3 hours per participant with breaks between experiments to provide proper rest to the participants. Sometimes the participants had small to moderate food intakes beforehand; most of the time they had none. Water was taken intermittently throughout experiments.

The EEG cap was placed on the participant's head and gel was inserted into the electrodes to activate their conductivity. After the impedance of the electrodes was checked and the rest of the equipment was tested, all electrical devices were switched off and the participant lay down ready to commence the experiment. At the end of the experiment, the cap was washed with a special liquid and dried properly before being used again. The availability of two caps allowed the experiments to run smoothly and efficiently throughout the two weeks. Participants used the same sized caps (medium) though we conveniently had access to small and large sized caps also.

\section{Signal Processing Aproach}

The EEG signal processing methodology and algorithms are comprised of the following steps:

1 Importing and preprocessing the data.

2 Calculate temporal Nyquist sampling frequencies.

3 Calculate the temporal power spectral densities (PSDt), for each of the 19 channels in windows of one (1) second.

4 Analyze the shape of the PSDt and derive quantitative measures based on the power of each frequency band in order to associate them with the participant's cognitive states. The data acquisition and spectral analysis software had specific parameters concerning sampling frequency,
Nyquist frequency, resolution and others. A summary of the frequency bands analyzed is provided in Table 1.

Table 1. Frequency Windows Analyzed

\begin{tabular}{|c|c|}
\hline Frequency Band & $\begin{array}{c}\text { Windows (Hz) } \\
\mathbf{f}_{\text {Low }}-\mathbf{f}_{\text {High }}\end{array}$ \\
\hline Theta & $4-6$ \\
\hline Alpha & $8-12$ \\
\hline Low Beta & $14-18$ \\
\hline High Beta & $20-26$ \\
\hline Low Gamma & $28-36$ \\
\hline High Gamma & $38-48$ \\
\hline
\end{tabular}

We use the following frequency bands over the PSDt functions to calculate the power of the signals in the specific ranges: Theta Power (TP: $4 \mathrm{~Hz}$ to $6 \mathrm{~Hz}$ ), Alpha Power (AP: $8 \mathrm{~Hz}$ to $12 \mathrm{~Hz}$ ), Low Beta Power (LBP: $14 \mathrm{~Hz}$ to $18 \mathrm{~Hz}$ ), High Beta Power (HBP: $20 \mathrm{~Hz}$ to $26 \mathrm{~Hz}$ ), Low Gamma Power (LGP: $28 \mathrm{~Hz}$ to $36 \mathrm{~Hz}$ ), and High Gamma Power (HGP: $38 \mathrm{~Hz}$ to $48 \mathrm{~Hz}$ ). Using these quantities, we define the maximum power and dominant power as follows

$$
\begin{array}{r}
P^{*}=\max \{T P, A P, L B P, H B P, L G P, H G P\} \\
\text { Maximum Power } \\
D F B=f \text { Band }\left(P^{*}\right) \\
\text { Dominant Frequency Band }
\end{array}
$$

where $\mathrm{P} *$ is the maximum power between the powers associated with every frequency band (TP, AP, LBP, HBP, LGP, HGP) and DFB is the Dominant Frequency Band in terms of power and is equal to $\mathrm{fBand}\left(\mathrm{P}^{*}\right)$ where $\mathrm{fBand}$ is the frequency band associated with $\mathrm{P}^{*}$.

Once the dominant band is determined for each window of duration 1 second, for each channel, then we produce:

1 Spatio temporal movies based on the DFB for each 1 s window and channel.

2 Comprehensive graphs for the DFB for all $1 \mathrm{~s}$ windows for all channels.

3 Statistical comparative analysis between modalities. 
For the first study we produced the spatial power spectral densities (PSDx), as well as the PSDt for the three modalities measured on the frontal area only and the reader is encouraged to review the results as described in detail in [11].

\section{Experimental Results}

The first set of graphs we present allows the evolution of the DFB per channel along a period of 400 seconds (6.66 minutes) to be seen. It is important to note that the first 10 seconds of all data was removed to eliminate the effects of the initialization process of recording and only 400 consecutive seconds after that were included for analysis.

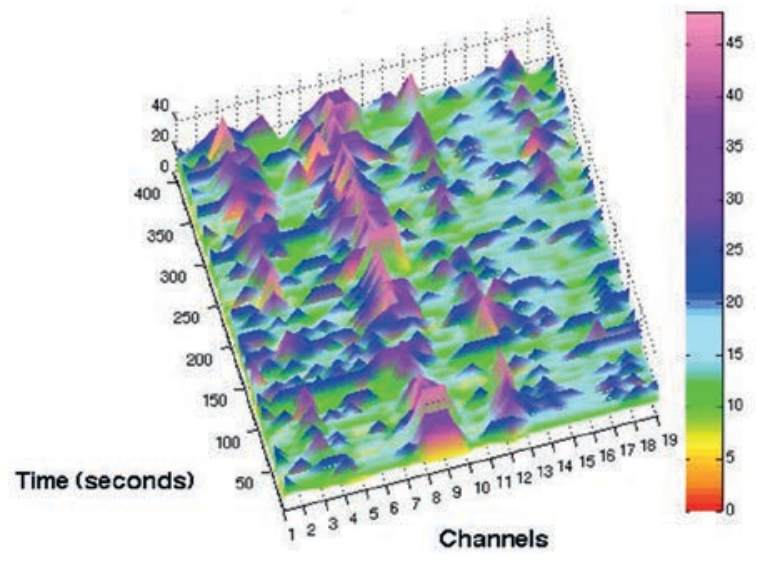

Figure 3. Shows the 19 channels EEG Dominant Frequency Band (DFB) for 400s for Participant one (1) in modality OEFL.

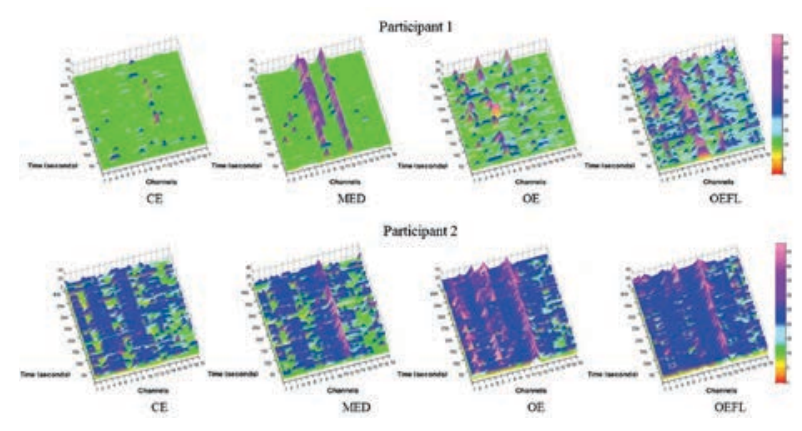

Figure 4. Shows the 19 channels EEG Dominant Frequency Band (DFB) for 400s for participant one (1) and two (2) in all modalities. Above: Participant 1 and Below: Participant 2 with CE, MED, OE, OEFL left to right for both participants.
We observe in Figure 3 that in a visual stimulus condition some channels like $3,4,8,9$ and 12 , for example, present abundant Low and High Gamma Frequency, while channels 14 to 19 are more dominated by Low and High Beta, together with Alpha. We also observe that the system is very dynamic in the way it shifts from frequency band to frequency band. These dynamics can be very well appreciated in the movies.

In Figure 4 we present the graphs for both participants in all modalities where we observe that there is a significant qualitative difference between modalities for each participant, although each participant shows different kinds of dynamics generally speaking. This is expected since every participant's brain is unique in configuration and the way it processes information about different cognitive states. Also, we know a priori that participant one (1) is a more advanced meditator than participant two (2), something we can also observe reflected in the graphs, since participant one has more of the Alpha frequencies present along time and modalities.

In general, the different modalities present the following characteristics concerning the dominant frequency band (DFB):

\section{For Participant 1}

- CE is predominantly in Alpha for all channels with significant presence of Low Beta for most channels at all times.

- OE is predominantly in Alpha though most channels, apart from 18 and 19, show occasional transitions into Beta and Gamma.

- OEFL is predominantly in High Beta, however, it is still dominated by Alpha and Low Beta with channels 4, 8, 9, and 10 showing abundant Low and High Gamma Frequency, while the rest of the channels are making frequent transitions between frequency bands mainly Alpha and Beta and occasionally some Gamma.

- MED is predominantly in Alpha, though channels 8 and 12 around the ears are showing abundant Gamma, presumably because of discomfort near that area of the head or some sensitivity to environmental noise. Apart from that, MED and $\mathrm{CE}$ are very similar in brain dynamics, although CE shows significant Low Beta for most channels at all times. 


\section{For Participant 2}

- CE is predominantly in High Beta for all channels with significant presence of Alpha and with some Low Beta and Theta for a few channels at different times.

- OE is predominantly in High Beta with a significant presence of Gamma in channels 2, 6, 8, and 12, and some Alpha and Low Beta for channels $16,17,18$, and 19 .

- OEFL is predominantly in High Beta, with some Gamma, particularly in channel 12 .

- MED shows a good mixture of Alpha and High Beta with some Gamma in channels 12.

In order to better appreciate the areas of the brain measured on the scalp, together with its associated frequency and transitions, we created a set of movies that gives great insight into the spatiotemporal dynamics associated with the different modalities and participant's brain dynamics. Due to the limitations of this paper we only display four frames of 1 second each of the movies of participant one (1) in two (2) modalities, CE and OEFL. The movies mimic the spatial brain map in Figure 1.

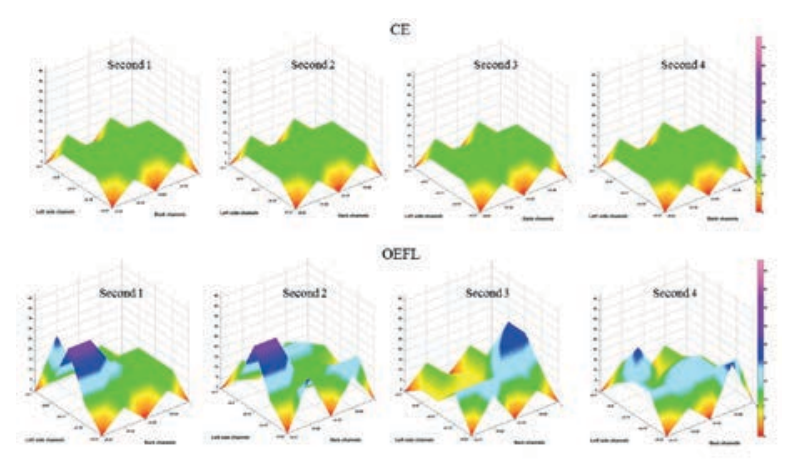

Figure 5. Shows the 19 channels EEG Dominant Frequency Band (DFB) plot of a matrix of $5 \times 5$ for four (4) consecutive seconds of participant one (1). Some channels are anchored in zero to mimic the brain map in Figure 1. Above: Modality CE and Below: Modality OEFL.

We clearly observe the difference between the two (2) modalities, which is very noticeable across the four frames displayed of 1 second each. We also observed different rotational and pulsation patterns, as well as the areas of the brain that were more active than others in the different modalities, which we conjecture are related to the nature of the cognitive states associated to them. This clearly requires more research, though it looks very promising.

Finally, we produced a very preliminary statistical analysis to show the difference between the cognitive states associated to each modality, by first calculating the difference between the matrices containing the data associated to each modality and then plotting the differences to compare them qualitatively. In Figure 5, we observe how close or far apart the modalities are from one another for both Participant 1 and 2, by observing how flat or bumpy the plot is respectively when we look at it as a field. We can easily tell that:

- CE is very similar to MED.

- MED is different than OE and this difference is similar to the difference between $\mathrm{CE}$ and $\mathrm{OE}$.

- OEFL is very different than CE, MED, and OE.

In order to have a quantitative measure for these differences we calculated box plots in different modalities as follows. First, we computed the mean for each 1s window for all channels (subscript c) by modalities and for each participant, MFAC(t), and we applied the box plot analysis to these two sets of data as shown in Figure 6.

$$
\operatorname{MFAC}(\mathrm{t})=\sum_{\mathrm{c}=1}^{19} \frac{\operatorname{DFB}(\mathrm{t})_{\mathrm{c}}}{19} ; \quad \forall \mathrm{t}=1,2, \ldots 400 \text {. }
$$

We can appreciate some interesting differences between participants and between modalities. For Participant 1 , the CE modality shows a median with a very small spread around $10 \mathrm{~Hz}$ indicating that the Alpha frequency band is dominating.

The OE modality follows with a slightly higher median also around $10 \mathrm{~Hz}$ and a larger spread. Then the MED modality shows a bigger median around $11.5 \mathrm{~Hz}$ with a slightly larger spread than $\mathrm{OE}$, and finally we observe the OEFL modality with a median close to $12 \mathrm{~Hz}$ and a significantly greater spread. This indicates that the probability distributions for the mean of each modality are more likely significantly different. Participant 2 shows different results for each modality. For the modalities of MED and CE we observe medians close to $11 \mathrm{~Hz}$ with quite a large spread. For the $\mathrm{OE}$ 
and OEFL modalities the medians are significantly larger, around $14 \mathrm{~Hz}$, with an even larger spread. This is definitely showing us a difference between modalities where CE and MED states are different than OEFL. OE deviates for Participant 1 to lower values than MED, something that may be showing us how well trained in embodying meditative states this participant is, even with her eyes open. This is further supported when we notice that overall, Participant 1 presents lower values than Participant 2 for the median for all modalities.

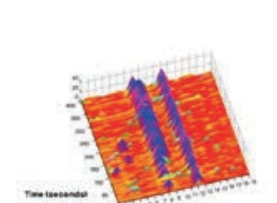

MED vs CE
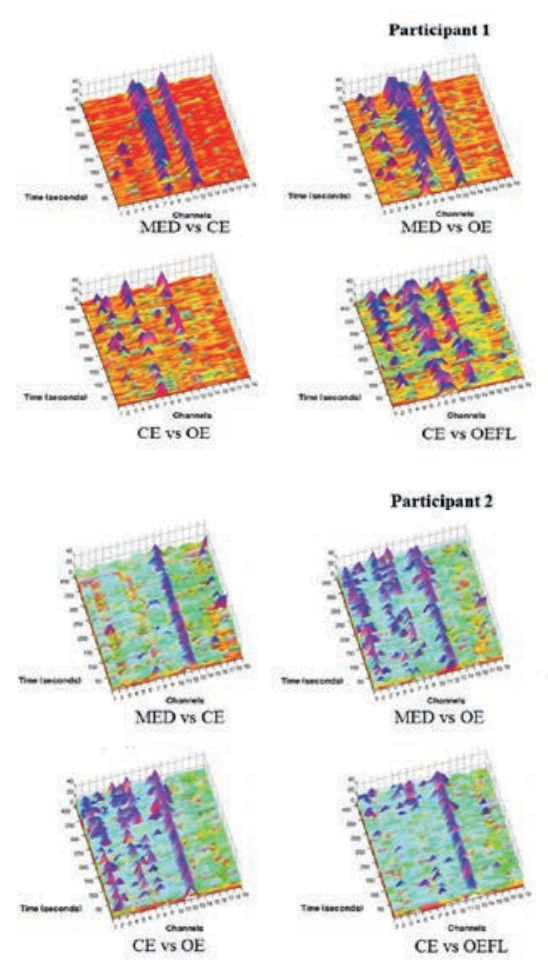

CE vs OEFL
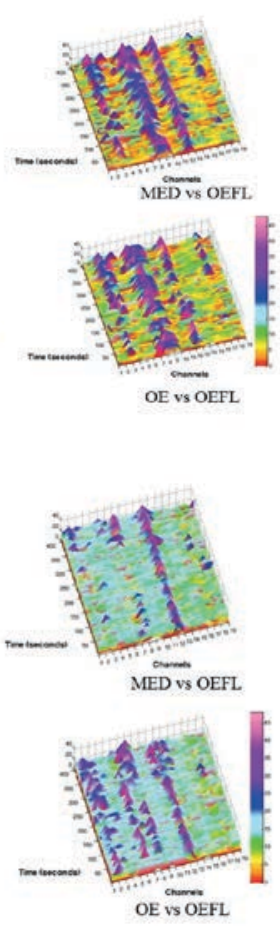

Figure 6. Shows a comparison between modalities by participants. Above: A set of six (6) graphs for Participant 1. Below: A set of six (6) graphs for Participant 2. The order in which they are displayed is from left to right showing the comparison between: (1) MED vs. CE, MED vs. OE, MED vs. OEFL for the upper row and (2) CE vs OE, CE vs. OEFL and OE vs. OEFL for the bottom row.
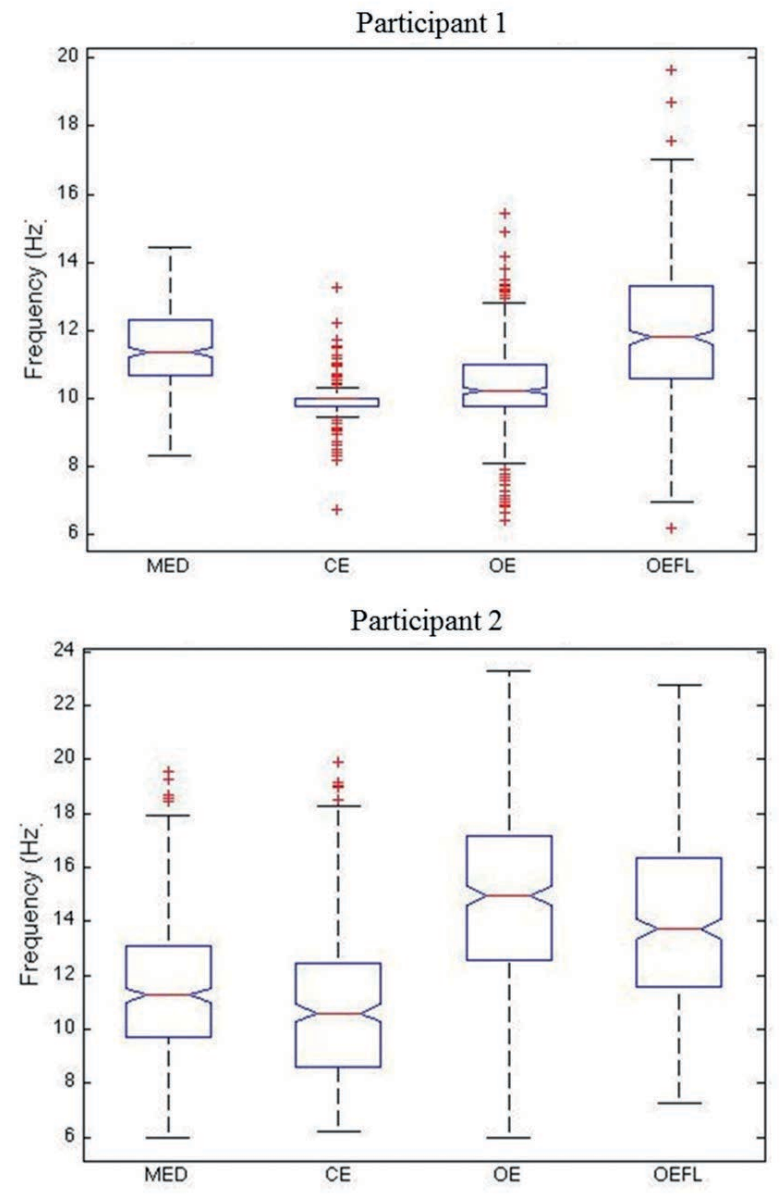

Figure 7. Boxplot analysis for the vectors of the Mean Frequency for all channels calculated for every $1 \mathrm{~s}$ windows, $M F A C(t)$, for all modalities for each participant. Above: results for Participant 1.

Below: results for Participant 2.

The second analysis we performed was based on the differences between modalities computed as follows

$$
\operatorname{DIFF}_{c}=\operatorname{abs}\left\{\operatorname{DFB}(t)_{j c}-\operatorname{DFB}(t)_{k c}\right\}
$$

for $k \neq j$ and $\forall c=1,2, \ldots 19$, where $k$ and $j$ are the different modalities MED, CE, OE, OEFL and therefore, $k=1,4 \& j=1,4$.

Note that when $k=1$ and $j=3$, for example, that DIFFc is the same as when $\mathrm{k}=3$ and $\mathrm{j}=1$, and therefore we use only one of the two alternatives.

After this calculation, we apply to $\operatorname{DIFF}(\mathrm{t})_{c}$ the same treatment as in equation (4) and we obtain the mean of the differences as follows 


$$
\operatorname{MDIFF}(\mathrm{t})=\sum_{\mathrm{c}=1}^{19} \frac{\operatorname{DIFF}(\mathrm{t})_{\mathrm{c}}}{19} ; \quad \forall \mathrm{t}=1,2, \ldots 400
$$

Next we apply the box plot analysis to $\operatorname{MDIFF}(\mathrm{t})$ for each pair of modalities as follows (see Table 2): MED vs. CE where $k=1$ and $j=2$; MED vs. $\mathrm{OE}$ where $\mathrm{k}=1$ and $\mathrm{j}=3$; MED vs. OEFL where $\mathrm{k}=1$ and $\mathrm{j}=4$; CE vs. OE where $\mathrm{k}=2$ and $\mathrm{j}=3$; CE vs. OEFL where $\mathrm{k}=2$ and $\mathrm{j}=4$; and $\mathrm{OE}$ vs. $\mathrm{OEFL}$ where $\mathrm{k}=3$ and $\mathrm{j}=4$.

Table 2. Modalities to Compare

\begin{tabular}{|l|l|l|l|l|l|}
\hline & $\mathbf{k}$ & 1 & 2 & 3 & 4 \\
\hline $\mathbf{j}$ & & MED & CE & OE & OEFL \\
\hline 1 & MED & - & vs. & vs. & vs. \\
\hline 2 & CE & - & - & vs. & vs. \\
\hline 3 & OE & - & - & - & vs. \\
\hline 4 & OEFL & - & - & - & - \\
\hline
\end{tabular}

Following, in Figure 7 we observe the results for the mean differences MDIFF( $\mathrm{t}$ ) between modalities when applying the box plots analysis. We can observe very similar results for Participant 1 , as already mentioned above in the previous analysis, basically:

- MED, CE, and OE present very significant differences from OEFL as expected since OEFL is a very busy and perhaps uncomfortable visual stimuli that could distract even the most masterful meditator.

- MED and CE show more similarities as expected since $\mathrm{CE}$ is a kind of meditative state. The difference between the two is associated with behavior of channels 8 and 12 near the ears, as discussed above.

- OE and CE also show great similarities, something that we can only attribute to the fact that Participant 1 is masterful enough to be in a meditative state while in OE.

- MED and OE present a slightly larger difference, something we attribute to the behavior of channels 8 and 12 near the ears in the MED modality.

For Participant 2 we also observe very similar results as already mentioned above in the previous analysis, basically:
- Most of the modalities show significantly different dynamics, apart from the modalities MED and $\mathrm{CE}$ that are slightly different from one another. All of the modalities are quite similar in their differences, showing a large spread when compared with one another and an associated median of around $7 \mathrm{~Hz}$ of difference, apart from the MED vs. CE comparison where the mean is around $5 \mathrm{~Hz}$ difference.
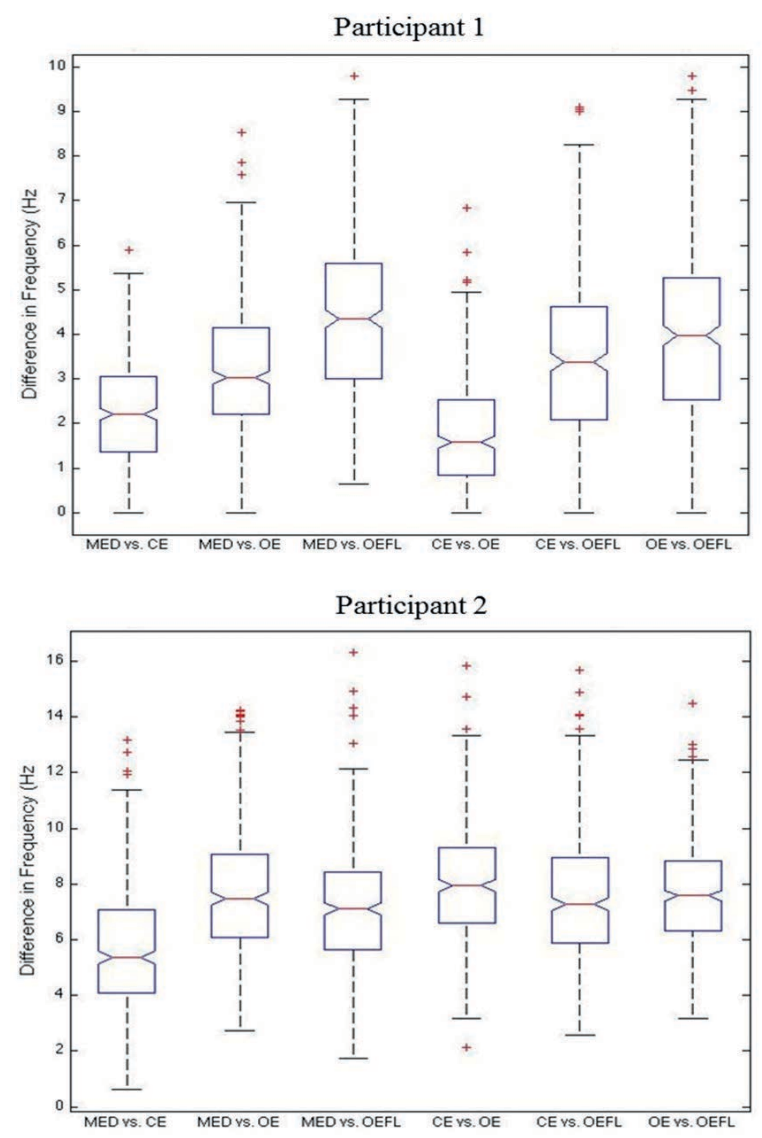

Figure 8. Boxplot analysis for the vectors of the Mean Frequency of the differences between modalities for all channels, calculated for every $1 \mathrm{~s}$ window, $\operatorname{MDIFF}(t)$, for each participant. Above: results for Participant 1. Below: results for Participant 2.

The last analysis we performed was a calculation of the norm or Euclidean distance (ED) on the vector $\operatorname{MDIFF}(\mathrm{t})$ which gave us a measure for the average distance between modalities. Following we can observe the results in Figure 8.

These measures confirm what the analysis, based on the box plots, has already shown. The reader is encouraged to verify these results with the above comments and analysis. 


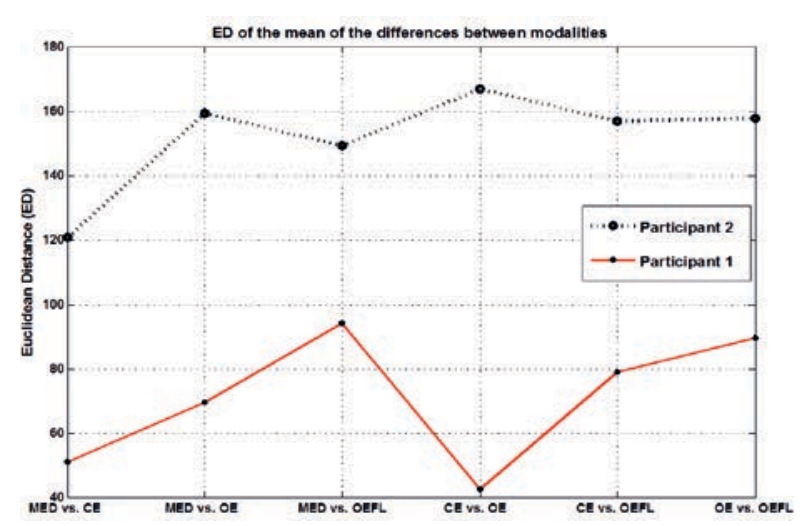

Figure 9. Euclidean Distance (ED) for the vectors of the Mean Frequency of the differences between modalities for all channels $M D I F F(t)$, calculated

for every $1 \mathrm{~s}$ window, for each participant.

We can clearly observe that the results of Participant 1 show significantly less distance between modalities than Participant 2. For Participant 1 OEFL is significantly different than $\mathrm{MED}, \mathrm{CE}$, and $\mathrm{OE}$, while for Participant 2 most modalities when compared are similar in their differences, apart from MED vs. CE modalities.

These are very interesting findings that when further tested and complemented with Hilbert analysis [23], may equip us with a very powerful methodology for future studies, when also complemented with brain movies and better classification algorithms, which can take in consideration the different aspects of spatio-temporal brain dynamics for different modalities.

At this stage, it is important to note that for the first study we obtained similar results overall concerning the differences between modalities and participants.

The following Figure 9 and Table 3 show some of the results that were obtained in the first study and the reader is again encouraged to revisit that study.

We can appreciate in Table 3 the values associated to the average slope $(\alpha)$ and the standard deviation (STD) of over all channels, for all windows and for each modality and participant. Since, our database is limited to only two (2) participants we are unable to do a systematic study with conclusive results. However, we can point out to some preliminary yet important illustrative observations. The CE modality seems to produce steeper slopes for both participants, while the OE and OEFL modali- ties show lower slopes. We observe more prominent changes between different modalities for Participant 2, while the $\mathrm{CE}$ and $\mathrm{OE}$ experiments demonstrate similar average slopes for Participant 1 . We also observed that the two (2) participants displayed different levels of tolerance to keep their eyes open for extended periods of time, as well as, the mastery to minimize artifacts. This together with the level of mastery in relaxation could be the cause for some of the differences we observe.

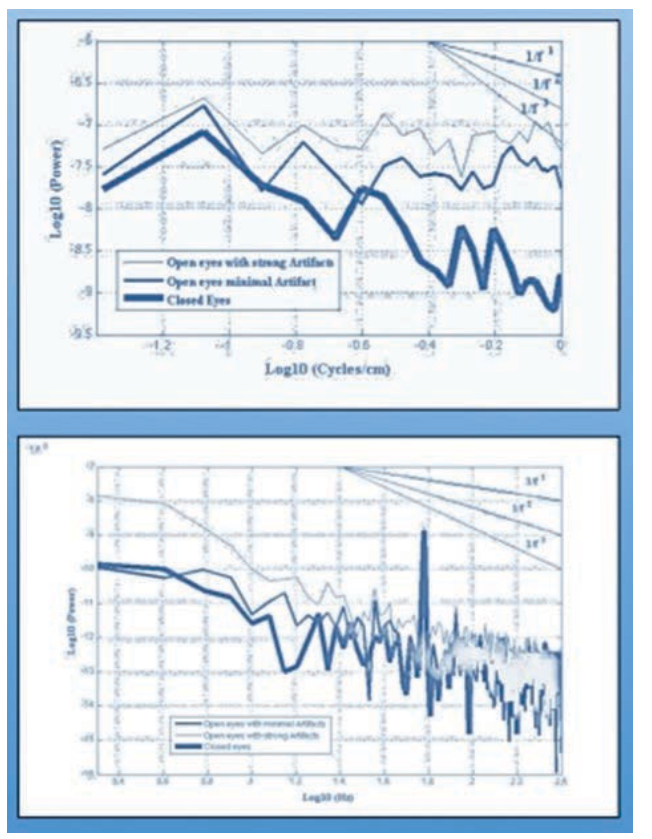

Figure 10. Top: Comparison of the spatial power spectral density functions (PSDx) at various experimental conditions averaged over the 48 electrodes. Bottom: temporal power spectral densities (PSDt) averaged over the 48 electrodes; experimental conditions: OE (minimal artifact),

OEFL (strong blinking artifact), and CE; for participant 2.

\section{Discussions}

The results that we have presented show a diverse set of tools and algorithms to understand brain dynamics both qualitatively and quantitatively. All of the measures, graphs and movies, provide valuable information concerning the dynamic transitions that the cortex displays along time and space across modalities for different participants. These results are showing us a direction towards finding better means in order to classify different brain cognitive states, however the work at hand is vast and far from complete. 
Table 3. Slope $(\alpha)$ and STD of the slope of PSDt*

\begin{tabular}{|c|c|c|c|c|c|c|}
\hline Participant & Closed Eyes & CE & Open Eyes & OE & Open Eyes & OEFL \\
\hline & Mean & STD & Mean & STD & Mean & STD \\
\hline 1 & -1.61 & 0.42 & -1.53 & 0.43 & -1.55 & 0.40 \\
\hline 2 & -1.81 & 0.41 & -1.59 & 0.37 & -1.45 & 0.36 \\
\hline
\end{tabular}

* Mean and standard deviation (STD) values were determined over 48 channels of the EEG array

There is a need to explore and apply new classification and pattern recognition methods for future more robust evaluation [10].

We have obtained very good results in terms of minimizing artifacts due to EMG, since the participants have been trained in how to achieve relaxed body states with minimal general body movement $[5,11]$. In the future we envision participants that train by watching movies or graphs giving biofeedback through which they can learn about themselves and their bodies. We also see the possibility of supporting the design of better equipment for EEG signal monitoring and measurement as part of the new technologies to appear concerning biofeedback systems.

We feel confident that this methodology will continue to contribute in allowing us to understand brain cognitive states subjectively and objectively, since we already have managed to show, though preliminary and only with two participants, the difference between modalities and participant's brain dynamics.

We have accomplished this work with the experience of previous studies in mind [7, 11, 20], in order to understand and gain more experience about the methodology and the difference between equipment. This study can be regarded as the continuation of previous studies showing consistency and new vistas for analysis, adding to our knowledge of brain dynamics and cognition. It would be appropriate, in future studies, to integrate this analysis to new data about functional interaction between brain areas. At this stage, we can state that for the condition where a flashlight was used as a visual stimulus, we observe more activity in the Gamma range in channels: (a) 3 and 4 associated with the frontal lobe, (b) 9 associated to the somatosensory cortex and (c) 8 and 12 associated to the temporal lobe. Also, the channels that are showing mainly low and high Beta together with some Alpha are: (a) 13 and
17 associated to the temporal lobe, (b) 14, 15 and 16 associated with the parietal lobe and (c) 18 and 19 associated with the occipital lobe.

All of this different activity is expected when compared to the CE modality, since the process of an intense visual stimulus involves two pathways that encompass the areas of the brain already mentioned. Also, these results could need more deep investigations in terms of artifact generation, even though we feel that these events were minimal due to training.

Generally speaking, the MINDO technologies would allow a much granular and refined spatial analysis and representation of brain dynamics with movies, provided that they are extended to encompass the whole scalp or at least larger areas via square arrays. The Mitsar 201 technology provided data collection for a larger area of the brain, however, unlike the MINDO technology, the spatial resolution was very poor for a more refined analysis and movie display. Both technologies would benefit with larger sample rates.

\section{Conclusions}

The results we present are in agreement with our previous study $[4,7,11,20]$ concerning the use of Power Spectrum Density (PSD) in order to discriminate between modalities and participants. Also, the brain movies created for this study, together with other methods for preliminary analysis, were inspired by previous studies on brain signal analysis towards a comprehensive methodology for the study and understanding of brain dynamics in the creation of knowledge and meaning $[14,15,16,17$, $18,19,21,23]$.

Our main conclusion can be summarized as follows:

- We successfully measured two (2) participants in four (4) different modalities and gathered 
enough data to do a robust analysis in order to compare brain dynamics in different cognitive states associated to the different modalities.

- We developed new types of graphs and movies that show spatio-temporal dynamics and transitions from and into different frequency bands measured on nineteen (19) different areas of the scalp. These movies allow us to better appreciate the differences between participants and modalities, and qualitatively discriminate with very good subjective accuracy the difference between participants and modalities and their associated brain dynamics.

- We analyzed quantitatively the data applying some mathematical transformations in order to produce comparative boxplots based on Mean Frequency Band per modality, per participant as well as, the Euclidean Distance (ED) measure calculated on the mean of the differences per modality and participants based on the Dominant Frequency Band per channel in every 1s window.

- We observed some important differences between a trained meditator and a very advanced one. Participant 2 is more diverse in frequency band transitions than Participant 1, presumably associated with different active and changing cognitive states. Participant 1 is more stable than Participant 2 in Alpha, across all modalities. All of these observations were somehow reflected, in both power spectrums (PSDt and PSDx) in the previous study. The PSDx was only possible to compute using the MINDO technology due to spatial resolution.

- Differences in modalities are smaller in the occipital and parietal regions of the scalp, something we can clearly observe in the graphs and particularly the movies. These movies were only produced with the data collected with the Mitsar 201 technology since it covered the whole scalp area regardless of poor spatial resolution

We foresee that this line of research could potentially help in improving the understanding of brain dynamics and therefore supporting to better understand what constitutes mental health. This could also lead to the development of better biofeedback systems and software to aid in new approaches to cognitive therapies and meditative practices, in order to unlock our spiritual potential towards a peaceful society.

\section{Acknowledgments}

This work is based on a collaborative effort between The Embassy of Peace in New Zealand, Brain Research Center, NCTU, Taiwan, and Kozmos Lab, USA. We express our gratitude to Dirk de Ridder, for his kind support with the EEG equipment and his advice on research requirements; Igor Meglinski, for his valuable input in the physics of light and its potential applications to biological processes; Samantha Ross, for her time and dedication to explain the operation of the EEG equipment and Colin Day, for providing a whole house for the Lab to fully operate for a period of three weeks. This work was also made possible by the invaluable support of Maria, Shahar, Kali, Carey and Sarah at The Embassy of Peace in New Zealand and the two voluntary participants, whom devoted their time and energy to the successful completion of this study.

Finally, we dedicate this work to the memory of our beloved Walter J. Freeman (1927-2016), a pioneer of brain research who has been a great source of inspiration for our work.

\section{References}

[1] W. J. Freeman and R. Quian Quiroga, Imaging Brain Function with EEG, New York: Springer, 2013

[2] W. J. Freeman, L. J. Rogers, M. D. Holmes, and D. L. Silbergeld, Spatial spectral analysis of human electrocorticograms including the alpha and gamma bands, J. Neurosci. Methods, vol. 95, pp. 111-21, 2000

[3] W. J. Freeman, M. D. Holmes, B. C. Burke, and S. Vanhatalo, Spatial spectra of scalp EEG and EMG from awake humans, Clin. Neurophysiol., vol. 114, no. 6, pp. 1053-1068, 2003

[4] W. J. Freeman and J. Zhai, Simulated power spectral density (PSD) of background electro-corticogram (ECoG), Cogn. Neurodyn., vol. 3, no. 1, pp. 97-103, 2009

[5] W. J. Freeman, C. Ramon, and M. D. Holmes, 1D spatial autocorrelation function of EEG: a sensitive assay for occult EMG, 16th Conf. Human Brain Mapping, \#881, 2010 
[6] G. Buzsaki, Rhythms of The Brain, New York: Oxford University Press, 2006

[7] R. Kozma, J. J. Davis, C.-T. Lin, L.-D. Liao and W. J. Freeman, Optimizing EEG/EMG signal to noise ratio at high spatial resolution, SfN Congress, \#586.12/NNN11, Nov. 9- 12, 2013, San Diego, CA, USA

[8] S. Pockett, G. E. J. Bold, and W. J. Freeman, EEG synchrony during a perceptual-cognitive task: Widespread phase synchrony at all frequencies, Clin. Neurophysiol., vol. 120, pp. 695-708, 2009

[9] Y. Ruiz, S. Pockett, W. J. Freeman, E. Gonzalez, and G. Li, A method to study global spatial patterns related to sensory perception in scalp EEG, J. Neurosci. Methods, vol. 191, pp. 110-118, 2010

[10] N. Kasabov and E. Capecci, Spiking neural network methodology for modelling, classification and understanding of EEG spatio-temporal data measuring cognitive processes, Information Sciences, vol. 294, pp. 565-575, 2015

[11] R. Kozma, J. J. J. Davis, C.-T. Lin, and W. J. Freeman. Spatio-Temporal EEG Pattern Extraction Using High-Density Scalp Arrays, IEEE World Congr. on Comp. Intel., 2016

[12] K. Pribram, The Form Within - My Point of View. Westport, CT: Prospecta Press, 2013

[13] Mitsar Brain Diagnostics Solutions, EEG Accessories:

Available:http://www.mitsar-medical.com/eegaccessories/ (last viewed 15 April 2016)

[14] J. J. Davis and R. Kozma, Analysis of phase relationship in ECoG using Hilbert transform and information theoretic measures, 2012 IJCNN, Bris., Australia, 10-15 June, 2012

[15] J. J. J. Davis, W. J. Freeman, and R. Kozma, Synchronized Minima in ECoG Power at Frequencies Between Beta-Gamma Oscillations Disclose Cortical Singularities in Cognition, Journal of Neuroscience and Neuroengineering, vol. 1, no. 1, pp. 1323, 2012

[16] J. J. Davis and R. Kozma, On the Invariance of Cortical Synchronization Measures Across a Broad Range of Frequencies, $20124^{\text {th }}$ iCAST

[17] J. J. Davis and R. Kozma, Creation of Knowledge and Meaning Manifested via Cortical Singularities in Cognition: Towards a Methodology to Understand Intentionality and Critical Behaviour in Neural Correlates of Awareness, 2013 IEEE Symp. Series on $\mathrm{CCMB}$
[18] J. J. J. Davis, W. J. Freeman, and R. Kozma, Neurophysiological evidence of the cognitive cycle and the emergence of awareness, 2013 iCAST -UMEDIA

[19] J. J. J. Davis, R. Ilin, R. Kozma, and M. H. Myers, Phase Cone Detection Optimization in EEG Data, IJCNN, 2014

[20] J. J. Davis, W. J. Freeman, R. Kozma, and C.-T. Lin, Model-based measurement of eeg data from linear high-density array (Poster Presentation), SfN Ann. Meet., 2014

[21] J. J. Davis and R. Kozma, Sensitivity analysis of Hilbert transform with band-pass FIR filters for robust brain computer interface, 2014 IEEE Symposium on CIBCI, Orlando, FL, 2014

[22] J. J. J. Davis, G. Gillett, and R. Kozma, Revisiting Brentano on Consciousness: Striking Correlations with Electrocorticogram Findings about the ActionPerception Cycle and the Emergence of Knowledge and Meaning, Mind and Matter, vol. 13, no. 1, pp. 45-69, 2015.

[23] J. J. J. Davis, R. Kozma, and W. J. Freeman, The Art of Encephalography to Understand and Discriminate Higher Cognitive Functions Visualizing Big Data on Brain Imaging using Brain Dynamics Movies, INNS Conf. on Big Data, Proc. Comp. Sci., Vol. XXX, pp. 1-8, 2015

[24] Mitsar Brain Diagnostics Solutions, Specifications: Available:http://www.mitsar-medical.com/eegmachine/eeg-amplifier-201/specific.html (last viewed 15 April 2016).

[25] J. J. J. Davis, The Brain of Melchizedek, M.S. thesis, Cog. Sci. Otago Uni., Dunedin, New Zealand, 2009

[26] J. M. Schwartz, H. P. Stapp, and M. Beauregard, Quantum physics in neuroscience and psychology: a neurophysical model of mind-brain interaction, Phil. Trans. R. Soc. B, doi:10.1098/rstb. 1598, 2004

[27] F. G. Echenhofer and M. M. Coombs, A Brief review of research and controversies in EEG Biofeedback and meditation, J. of Trans. Psych., vol. 19, no. 2, 1987

[28] A. Kasamatsu and H. Tomio, An electroencephalographic study on the zen meditation (zazen), Folia Psychiatrica et Neurologica Japonica, vol. 20, no. 4, 1966

[29] J. H. Austin, Zen and The Brain,Cambridge, MASS: The MIT Press, 1999 


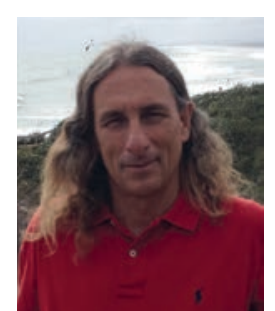

Jeffrey Jonathan (Joshua) Davis: together with a great love for surfing, Joshua is experienced as a decision analyst and strategic planner for banks, oil companies, consulting firms and family business. He lectured for several years in the fields of systems thinking, computer simulation, chaos theory, fractal geometry, decision making and systems dynamics. From 1994 onwards, after a set of meaningful spiritual experiences, he spent many years travelling as an Ambassador of Peace around the world. He also has a deep love for Jazz having learned the harmonica, guitar and the saxophone. Since 1998 he has worked in research concerning Decision Making and Consciousness and published a thesis, "The Brain of Melchizedek, A Cognitive Neuroscience Approach to Spirituality". More recently, he has been researching in close collaboration with Grant Gillett, Robert Kozma, Walter Freeman and Paul Werbos in the areas of cognitive neuroscience, philosophy, quantum physics and the biophysics of peace.

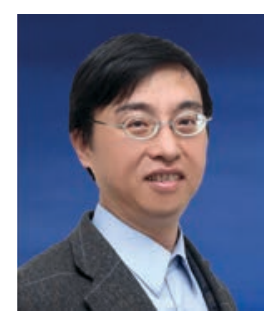

Dr. Chin-Teng Lin received the B.S. degree from National Chiao-Tung University (NCTU), Taiwan in 1986, and the Master and Ph.D. degree in electrical engineering from Purdue University, USA in 1989 and 1992, respectively. He is currently the Distinguished Professor of Faculty of Engineering and Information Technology, University of Technology Sydney, and Lifelong Chair Professorship of NCTU. Dr. Lin also own International Faculty of University of California at San-Diego (UCSD), and Honorary Professorship of University of Nottingham. Dr. Lin was elevated to be an IEEE Fellow for his contributions to biologically inspired information systems in 2005, and was elevated International Fuzzy Systems Association (IFSA) Fellow in 2012. Dr. Lin received the IEEE Fuzzy Systems Pioneer Award in 2017, Outstanding Achievement Award by Asia Pacific Neural Network Assembly in 2013, Outstanding Electrical and Computer Engineer, Purdue University in 2011, and Merit National Science Council Research Fellow Award, Taiwan in 2009. He served as the Editor-in-chief of IEEE Transactions on Fuzzy Systems from 2011 to 2016. He also served on the Board of Governors at IEEE Circuits and Systems (CAS) Society in 2005-2008, IEEE Systems, Man, Cybernetics (SMC) Society in 2003-2005, IEEE Computational Intelligence Society (CIS) in 2008-2010, and Chair of IEEE Taipei Section in 2009-2010. Dr. Lin is the Distinguished Lecturer of IEEE CAS Society from 2003 to 2005, and CIS Society from 2015-2017. He served as the Deputy Editor-in-Chief of IEEE Transactions on Circuits and Systems-II in 2006-2008. Dr. Lin was the Program Chair of IEEE International Conference on Systems, Man, and Cybernetics in 2005 and General Chair of 2011 IEEE International Conference on Fuzzy Systems. Dr. Lin is the coauthor of Neural Fuzzy Systems (PrenticeHall), and the author of Neural Fuzzy Control Systems with Structure and Parameter Learning (World Scientific). He has published more than 220 journal papers and 97 patents $(\mathrm{H}-$ index: 56) in the areas of computational intelligence, fuzzy neural networks, natural cognition, brain-computer interface, intelligent system, multimedia information processing, machine learning, robotics, and intelligent sensing and control, including approximately 107 IEEE journal papers.

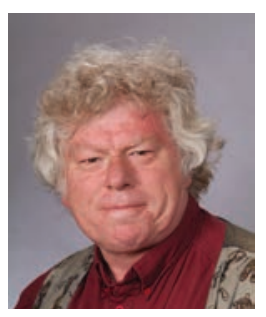

MBChB (equivalent to U.S. MD); MSc(Psychology); D.Phil (Oxon); FRACS (equivalent to U.S. Board Certification in Neurosurgery); FRS NZ.

Grant Gillett is a qualified neurosurgeon and practised until 2006. He also has a D.Phil (Oxon) and was a fellow in philosophy at Magdalen College, Oxford. He is now a Professor of Medical Ethics at the Bioethics Centre of the Otago University Medical School, and a fellow of the Royal society. He is author of The Mind and its Discontents (2009), Subjectivity and Being Somebody: human identity and neuroethics. (2008), Bioethics in the clinic: Hippocratic reflections (2004) and over 350 articles in medical ethics, philosophy, bioethics, the philosophy of psychiatry and neuroscience.

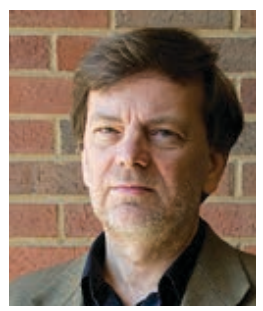

Dr. Robert Kozma holds a Ph.D. in Physics (Delft University of Technology, The Netherlands, 1992), M.Sc. in Mathematics (with honors, Eotvos University, Budapest, Hungary, 1988), and Diploma in Power Engineering (with distinction, Moscow Institute of Energy, Russia, 1982). He joined the Computer Science Department of the University of Massachusetts Amherst as Visiting Professor in 2016. He is Professor of Mathematics at the University of Memphis, where he has been Associate and Full Professor of Computer Science since he joined the ranks of faculty in 2000. Professor Kozma is Fellow of IEEE, Fellow of International Neural Network Society (INNS). He is President-Elect of INNS (2016), recipient of INNS Gabor Award (2011), and has been NRC Senior Fellow (2006-2008). He is on the Governing Board of IEEE SMC Society (2016-2018).

Professor Kozma's research focuses on developing novel artificial intelligent system motivated by the operation of brains. He conducts research on neural networks, cellular automata, random graph theory, machine learning, knowledge acquisition, computational neuroscience, neurally-inspired computing, brain modeling, processing massive brain imaging data, brain-computer interfaces, and autonomous decision support systems. 